\title{
Dipole-Dipole Broadening in the Selective Reflection of an Intense Laser Beam from the Interface between a Transparent Dielectric and a Dense Resonance Gas
}

\author{
A. A. Bobrov ${ }^{a, ~ *}$, S. A. Saakyan ${ }^{a, b}$, V. A. Sautenkov ${ }^{a, c}$, and B. B. Zelener ${ }^{a, d, e}$ \\ ${ }^{a}$ Joint Institute for High Temperatures, Russian Academy of Sciences, Moscow, 127412 Russia \\ ${ }^{b}$ National Research University Higher School of Economics, Moscow, 101000 Russia \\ ${ }^{c}$ Lebedev Physical Institute, Russian Academy of Sciences, Moscow, 119991 Russia \\ ${ }^{d}$ National Research University Moscow Power Engineering Institute, Moscow, 111250 Russia \\ ${ }^{e}$ National Research Nuclear University MEPhI (Moscow Engineering Physics Institute), Moscow, 115409 Russia \\ *e-mail:abobrov@inbox.ru
}

Received September 23, 2021; revised September 30, 2021; accepted October 4, 2021

\begin{abstract}
The dipole-dipole broadening of the spectrum of the selective reflection of intense resonance light from the interface between a transparent dielectric and a gas of the natural mixture of Rb isotopes has been studied experimentally. The case of a high gas density where the Doppler broadening can be neglected has been investigated. It has been shown that dipole-dipole broadening is reduced with increasing the number density of excited atoms. When the laser beam intensity is much higher than the saturation intensity of a resonance transition, a significant broadening due to the very high laser beam intensity has not been observed in the reflection spectrum from the transparent dielectric/gas interface. The observed intensity dependence of the spectral width has been explained by the quenching collisions of the excited atoms with the interface.
\end{abstract}

DOI: $10.1134 / \mathrm{S} 0021364021210086$

In gaseous media, the spectral width of a resonant atomic transition is significantly increased by the dipole-dipole interaction between identical atoms, one of which is in the ground state and the other is in an excited state $[1,2]$. The dipole-dipole broadening $\Gamma$ in a weakly excited medium is proportional to the density and can be expressed as

$$
\Gamma=K N=\frac{2 \alpha d^{2} N}{\hbar},
$$

where $d$ is the dipole moment of the transition, $N$ is the number density of atoms, and $\alpha$ is the coefficient about unity. Dipole-dipole broadening in dense gaseous media, which are studied in this work, is much larger than Doppler broadening.

The population of the excited state cannot be neglected in the case of irradiation of the gas of resonant atoms by an intense laser beam. A decrease in the population of the ground state should generally result in a decrease in dipole-dipole broadening. This counterintuitive phenomenon of the narrowing of the spectral line in a dense resonant gaseous medium with an increase in the radiation intensity was observed experimentally for the first time in [3]. To study the resonant interaction, the selective reflection technique, which allows one to analyze the spectrum of the strongly absorbing dense medium, was used in [3]. To achieve a significant population of the excited state of the absorbing gaseous medium, an intense laser pump beam far detuned from the resonance was used in [3]. The selective reflection spectrum was obtained with a weak probe beam scanned near the resonance. The dipole-dipole broadening for the gas of two-level atoms with a significant population of the excited state can be estimated by the formula [4] $\Gamma=$ $K\left(g_{\mathrm{g}} / g_{\mathrm{e}}\right)^{1 / 2} N_{\mathrm{g}}$, where $N_{\mathrm{g}}$ is the number density of atoms in the ground state, and $g_{\mathrm{g}}$ and $g_{\mathrm{e}}$ are the statistical weights of the ground and excited states, respectively. We note that the narrowing of the selective reflection resonance was observed only for dense media [5].

It is interesting to study the selective reflection spectrum of a single laser beam that simultaneously saturates the gas and forms a reflection signal. In this case, a decrease in dipole-dipole broadening at a high radiation intensity competes with an increase in the field broadening. The reflection of saturating radiation from a rarefied gas $[6,7]$ and the reflection of low-intensity radiation from a dense gas [8-10] were studied earlier. However, the selective reflection of intense radiation from the interface between a trans- 


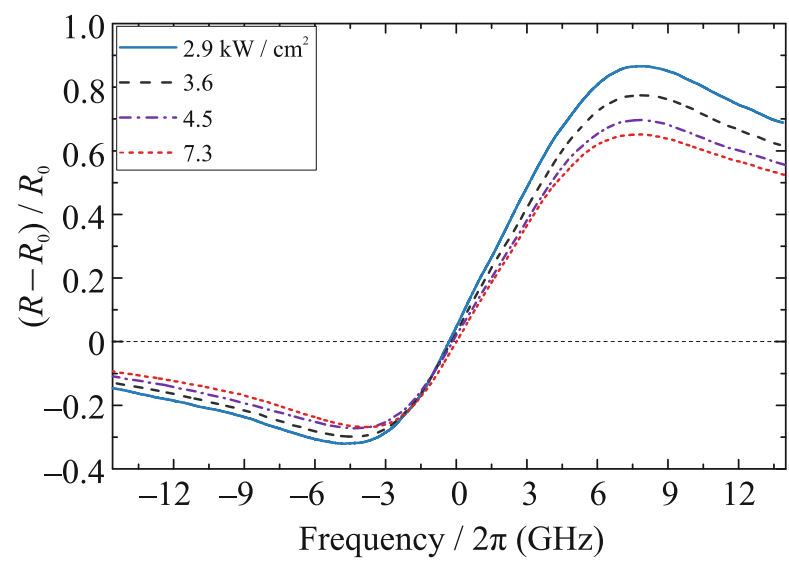

Fig. 1. (Color online) Relative coefficient of selective reflection for the intensities $I=2.9,3.6,4.5$, and $7.3 \mathrm{~kW} / \mathrm{cm}^{2}$ versus the frequency of incident radiation.

parent dielectric and a dense gaseous medium has not yet been studied. The aim of this work is to fill this gap.

We previously studied the selective reflection of a laser beam of moderate intensity, up to the saturation intensity [11]. A linear decrease in the spectral width at low intensities was observed in [11], as well as in [3]. The dependence of the width at maximum studied intensities began to deviate from the linear one. In this work, we continue to study the selective reflection spectrum in the strong saturation region.

The experimental procedure was described in detail in [11]; here we only briefly describe the experiment. The laser beam with a wavelength of $\lambda=780 \mathrm{~nm}$ was almost normally incident on the window of a cell filled with a gas of the natural mixture of $\mathrm{Rb}$ isotopes with a density of $8 \times 10^{16} \mathrm{~cm}^{-3}$ at a temperature of $350^{\circ} \mathrm{C}$. The beam frequency was scanned near the resonance frequency of the $5 S_{1 / 2}(F=3)-5 P_{3 / 2}\left(F^{\prime}=4\right)$ transition in ${ }^{85} \mathrm{Rb}$, which has a spontaneous decay rate of $2 \pi \times 6 \mathrm{MHz}$.

According to [12, 13], the coefficient in Eq. (1) for this transition can be estimated as $K / 2 \pi=(1.1 \pm$ $0.17) \times 10^{-7} \mathrm{~Hz} \mathrm{~cm}^{3}$, which corresponds to the spectral width $\Gamma / 2 \pi=(8.8 \pm 1.5) \mathrm{GHz}$. Correspondingly, the Doppler broadening can be estimated as $\Delta \omega_{\mathrm{D}} \approx 0.1 \Gamma$. It should be noted that the concentration of $\mathrm{Rb}_{2}$ molecules at this temperature is insignificant [14]; furthermore, the spectra of $\mathrm{Rb}$ atoms and $\mathrm{Rb}_{2}$ molecules do not overlap $[15,16]$.

Figure 1 shows the resulting frequency dependences of the relative reflection coefficient $\left(R-R_{0}\right) / R_{0}$, where $R_{0}=8.5 \%$ is the reflection coefficient of the window material. The relative coefficient was measured for the selective reflection of incident radiation from the inner surface of the window of the cell for various intensities $I$ of the laser beam. Because of significant dipole-dipole broadening, hyperfine

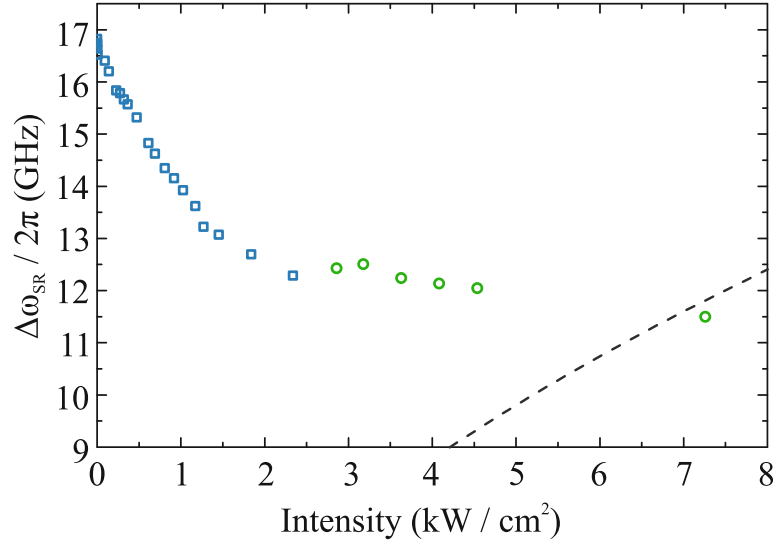

Fig. 2. (Color online) Width of the selective reflection spectrum versus the intensity of $780-\mathrm{nm}$ radiation. Squares and circles are the experimental data taken from [11] and obtained in this work, respectively. The line is the Rabi frequency for the $5 S_{1 / 2}-5 P_{3 / 2}$ transition in ${ }^{85} \mathrm{Rb}$.

components were not resolved in the reflection spectrum. The frequencies in Fig. 1 are measured from the frequency of the $5 S_{1 / 2}(F=3)-5 P_{3 / 2}\left(F^{\prime}=4\right)$ transition in ${ }^{85} \mathrm{Rb}$.

The width of the selective reflection spectrum $\Delta \omega_{\mathrm{SR}}$ was determined as the difference between the frequencies corresponding to the minimum and maximum values of the reflection coefficient. Figure 2 shows the dependence of the spectral width on the radiation intensity.

The spectral width at low intensities decreases linearly with increasing intensity because of a decrease in dipole-dipole broadening, as observed in previous works. Then, the width becomes constant owing to the saturation of the transition. It was expected that the width of the selective reflection spectrum at intensities much higher than the transition saturation intensity would be determined by the field broadening, but the field broadening was not observed in the experiment. The intensity dependence of the Rabi frequency $\Omega=d E / \hbar$, where $E$ is the field amplitude, for the $5 S_{1 / 2}-5 P_{3 / 2}$ transition in ${ }^{85} \mathrm{Rb}$ is shown in Fig. 2 for comparison. It is seen that the detected spectral width is smaller than the Rabi frequency for the strongest transition at the maximum used intensity.

The observed effect cannot yet be explained theoretically. The existing theoretical approaches $[6,7]$ to the selective reflection of the strong field were developed for the case of a low density gas. A self-consistent theory of selective reflection from the dense gas was developed in [9], but only the case of a weak field was considered. Only numerical calculations were performed for the case of a strong field and a dense medium [17]. The discussion of the possibility of developing a complete self-consistent theory of selective reflection of the strong field from the interface 
between the dielectric and dense gas is beyond the scope of this brief letter, but the observed effect of saturation in the reflection spectrum can be qualitatively explained as follows.

For simplicity, we consider the selective reflection of intense radiation from the interface between the transparent dielectric and dense gas of two-level atoms. As known [6,7], a selective reflection signal is formed by an induced dipole moment in a thin layer of the gas near the interface. Atoms moving toward the interface and from the interface deeper inside the gas make significantly different contributions to the observed reflection signal.

Atoms moving toward the interface are subjected to the radiation field for a time much longer than the lifetime of the excited state. The optical transition in these atoms in the strong field is saturated; consequently, the contribution to the induced dipole moment from atoms moving toward the interface should vanish at radiation intensity much higher than the transition saturation intensity.

On the contrary, atoms moving from the interface are in the ground state immediately after collision with the interface and make the decisive contribution to the induced dipole moment and, thereby, to the formation of the reflection signal. On one hand, the spectrum should be free of field broadening because the transition in these atoms is not saturated owing to collision with the interface. On the other hand, atoms moving in different directions make the same contribution to the dipole-dipole broadening of the reflection signal because it is quasistatic [18]. Since the transition in atoms moving toward the interface is saturated, this saturation should affect the contribution of these atoms to the dipole-dipole broadening of the transition. It is noteworthy that we consider the case of a dense gas, where the Doppler broadening can be neglected. The motion of atoms in our model results only in collisions of atoms with the interface.

According to the above assumptions, to qualitatively describe the experimental results, we represent the width of the reflection spectrum as the sum of dipole-dipole and intensity-independent broadenings (in the discussed experiments, the main contribution to the latter comes from the unresolved hyperfine structure):

$$
\Delta \omega_{\mathrm{SR}}=\Gamma+\Gamma_{0} .
$$

Taking into account the population of the ground state within the two-level model [19], we obtain the dependence of dipole-dipole broadening on the intensity in the form

$$
\Gamma=K N\left(1-\frac{1}{2} \frac{\Omega^{2}}{\gamma_{2}\left(\gamma_{12}+\frac{\Omega^{2}}{\gamma_{2}}\right)}\right),
$$

where $\gamma_{2}$ and $\gamma_{12}$ are the longitudinal and transverse relaxation rates, respectively.

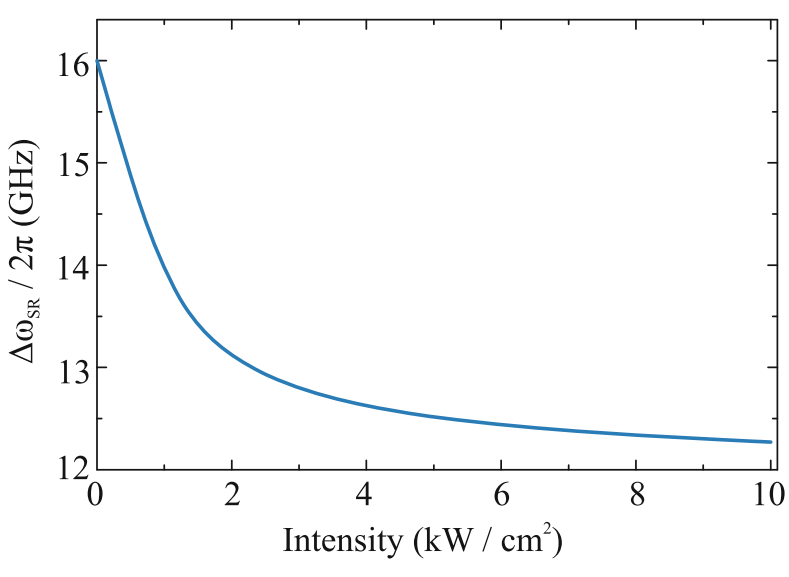

Fig. 3. (Color online) Width of the selective reflection spectrum calculated by Eqs. (2) and (3).

Figure 3 shows the width of the selective reflection spectrum calculated by Eq. (2), where dipole-dipole broadening was evaluated by Eq. (3) with the parameters $N=8 \times 10^{16} \mathrm{~cm}^{-3}, K / 2 \pi=(1.1 \pm 0.17) \times 10^{-7} \mathrm{~Hz} \mathrm{~cm}^{3}$, $\gamma_{2}=2 \pi \times 2 \mathrm{GHz}, \gamma_{12}=\Gamma_{0}=2 \pi \times 8 \mathrm{GHz}$, and the Rabi frequency $\Omega$ calculated with the dipole moment for the $5 S_{1 / 2}-5 P_{3 / 2}$ transition in ${ }^{85} \mathrm{Rb}$. It is seen that the width calculated by Eqs. (2) and (3) qualitatively reproduces the experimental dependence shown in Fig. 2.

To summarize, dipole-dipole broadening in the case of the selective reflection of the intense saturating laser beam from the interface between a transparent dielectric and a resonance gas has been studied for the first time. It has been found that the reflection spectrum is free of the field broadening even at very high intensities. This effect has been qualitatively explained by the quenching of the excitation of atoms at collisions with the interface. For the quantitative description of the experimental result, it is necessary to develop a selective reflection theory, which should involve both the quantum description of the transition saturation and the self-consistent description of the field damping in the dense medium, i.e., the spatial dispersion, and, in addition, should take into account hyperfine splitting. Radiation trapping and nonradiative excitation transfer in a dense medium can also affect the spatial distribution of excited atoms and, therefore, the observed reflection spectrum [20]. We emphasize that the medium at radiation intensities about and above the saturation intensity is nonlinear and the dielectric/gas interface can be considered as a nonlinear beam splitter; in this case, the quantum description of the radiation field can be required [21, 22].

\section{FUNDING}

This work was supported by the Ministry of Science and Higher Education of the Russian Federation (agreement 
no. 075-15-2020-785 on September 23, 2020, with the Joint Institute for High Temperatures, Russian Academy of Sciences).

\section{CONFLICT OF INTEREST}

The authors declare that they have no conflicts of interest.

\section{OPEN ACCESS}

This article is licensed under a Creative Commons Attribution 4.0 International License, which permits use, sharing, adaptation, distribution and reproduction in any medium or format, as long as you give appropriate credit to the original author(s) and the source, provide a link to the Creative Commons license, and indicate if changes were made. The images or other third party material in this article are included in the article's Creative Commons license, unless indicated otherwise in a credit line to the material. If material is not included in the article's Creative Commons license and your intended use is not permitted by statutory regulation or exceeds the permitted use, you will need to obtain permission directly from the copyright holder. To view a copy of this license, visit http://creativecommons.org/licenses/by/4.0/.

\section{REFERENCES}

1. E. L. Lewis, Phys. Rep. 58, 1 (1980).

2. A. A. Zabolotskii, J. Exp. Theor. Phys. 131, 520 (2020).

3. V. A. Sautenkov, H. van Kampen, E. R. Eliel, and J. P. Woerdman, Phys. Rev. Lett. 77, 3327 (1996).

4. H. van Kampen, V. A. Sautenkov, C. J. C. Smeets, E. R. Eliel, and J. P. Woerdman, Phys. Rev. A 59, 271 (1999).

5. V. A. Sautenkov, Laser Phys. Lett. 8, 771 (2011).

6. G. Nienhuis, F. Schuller, and M. Ducloy, Phys. Rev. A 38, 5197 (1988).
7. T. A. Vartanyan, Sov. Phys. JETP 61, 674 (1985).

8. P. Wang, A. Gallagher, and J. Cooper, Phys. Rev. A 56, 1598 (1997).

9. A. Badalyan, V. Chaltykyan, G. Grigoryan, A. Papoyan, S. Shmavonyan, and M. Movsessian, Eur. Phys. J. D 37, 157 (2006).

10. A. S. Kuraptsev, I. M. Sokolov, and Ya. A. Fofanov, Int. J. Mod. Phys. Conf. Ser. 41, 1660141 (2016).

11. V. A. Sautenkov, S. A. Saakyan, and B. B. Zelener, J. Russ. Laser Res. 42, 405 (2021).

12. C. B. Alcock, V. P. Itkin, and M. K. Horrigan, Can. Metall. Q 23, 309 (1984).

13. R. Kondo, S. Tojo, T. Fujimoto, and M. Hasuo, Phys. Rev. A 73, 062504 (2006).

14. M. Rakic and G. Pichler, J. Quant. Spectrosc. Radiat. Transfer 208, 39 (2018).

15. H. Chen, V. A. Sautenkov, P. S. Hsu, G. R. Welch, Y. V. Rostovtsev, and M. O. Scully, J. Mod. Opt. 52, 2373 (2005).

16. V. A. Sautenkov, S. A. Saakyan, A. M. Akulshin, M. A. Gubin, V. N. Kuliasov, and B. B. Zelener, J. Russ. Laser Res. 34, 375 (2013).

17. J. Guo, J. Cooper, A. Gallagher, and M. Lewenstein, Opt. Commun. 110, 732 (1994).

18. J. A. Leegwater and S. Mukamel, Phys. Rev. A 49, 146 (1994).

19. S. Stenholm, Foundations of Laser Spectroscopy (Wiley, New York, 1984).

20. Y. A. Fofanov, I. M. Sokolov, R. Kaiser, and W. Guerin, Phys. Rev. A 104, 023705 (2021).

21. A. V. Belinsky and M. Kh. Shulman, Phys. Usp. 57, 1022 (2014).

22. Ya. Fofanov, EPJ Web of Conf. 220, 01004 (2019).

Translated by $R$. Tyapaev 